\title{
Outcomes of laryngectomy in elderly patients with laryngeal carcinoma
}

\author{
R.J. Shi ${ }^{1}$, C.Z. Xu ${ }^{1}$, C.P. Zhang ${ }^{2}$, C.Y. Jiang ${ }^{1}$, Y.Y. Sun ${ }^{1}$, X.J. Yan ${ }^{1}$ and \\ P.H. Wang ${ }^{1}$ \\ ${ }^{1}$ Department of Otorhinolaryngology, \\ Shanghai Ninth People's Hospital Affiliated with the Shanghai Jiao Tong \\ University School of Medicine, Shanghai, China \\ ${ }^{2}$ Department of Oral \& Maxillofacial-Head \& Neck Oncology, \\ Shanghai Ninth People's Hospital Affiliated with the Shanghai Jiao Tong \\ University School of Medicine, Shanghai, China \\ Corresponding author: C.P. Zhang \\ E-mail: chenpingzhangcn@163.com
}

Genet. Mol. Res. 13 (1): 1955-1963 (2014)

Received June 25, 2013

Accepted November 1, 2013

Published March 17, 2014

DOI http://dx.doi.org/10.4238/2014.March.17.23

\begin{abstract}
Laryngeal carcinoma is a common tumor of the head and neck region. This study aimed to examine the outcomes of laryngectomy in elderly patients with laryngeal carcinoma. One-hundred twenty-two patients (male, 117; female, 5) aged 60 years or older (range, 60-94 years) who underwent laryngectomy between 1996 and 2010 were included. All patients were diagnosed with squamous cell carcinoma of the larynx, and 95 patients $(77.9 \%$ ) had additional concurrent diseases. Tumors were staged according to the TNM categories of the American Joint Committee on Cancer 2002 criteria; there were 16 stage-I, 24 stage-II, 52 stage-III, and 30 stage-IV cases. With regard to treatment modalities, 10 patients underwent transoral laser laryngectomy, 25 underwent partial laryngectomy, and 87 underwent total laryngectomy. When necessary, neck dissection was performed according to the Dalian criteria set in 2004 (a Chinese standard). Of the 122 cases, there were 114 cases of grade I (93.4\%), 5 cases of grade II, and 3 cases of grade
\end{abstract}


III (pharyngeal fistula in 2 cases recovered after 2 weeks of care) wound healing. No significant differences were observed in the occurrence or severity of comorbidities. The 1-, 3-, and 5-year actuarial survival rates were $97.5 \%(119 / 122), 84.4 \%(92 / 109)$, and $68.4 \%(67 / 98)$, respectively. Age alone should not be used to determine treatment options for elderly patients with squamous cell carcinoma. Presuming that careful pre-treatment evaluations are performed, laryngectomy is a key method for elderly patients with laryngeal carcinoma.

Key words: Laryngeal carcinoma; Laryngectomy; Squamous cell; Elderly

\section{INTRODUCTION}

In China, laryngeal carcinoma is one of the most common cancers, second only to nasopharyngeal carcinoma among cancers of the head and neck, and in some regions (Peking and Tianjin, not including Shanghai), the incidence of laryngeal carcinoma is the highest among tumors of the head and neck (Yang et al., 2005). Because significant progress has been made in geriatric health care, elderly patients with laryngeal carcinoma could potentially have a longer life expectancy than was previously possible. However, elderly patients with laryngeal carcinoma may have co-existing medical problems, and their natural life expectancy may be counted in years rather than decades; therefore, surgeons are increasingly required to perform all variations of laryngectomy in the elderly. Various retrospective studies have shown that radical surgery can be performed safely in elderly patients in the absence of severe concomitant morbidities (Barzan et al., 1990; Kowalski et al., 1994; Clayman et al., 1998; Derks et al., 2003). However, several studies showed that complications of head and neck surgeries increased significantly in elderly patients, suggesting that standard treatments for laryngeal carcinoma are not suitable for elderly patients (Greenfield et al., 1987; Bhattacharyya and Fried, 2001; Peters et al., 2011). Because data on the survival of elderly patients with laryngeal carcinoma are limited and conflicting, otolaryngologists can be faced with therapeutic dilemmas. The present study was conducted to determine whether elderly patients aged 60 years or older who were diagnosed with laryngeal carcinoma experienced substantially prolonged survival after surgical therapy.

\section{MATERIAL AND METHODS}

\section{Patients}

Details on the patients for the current study were extracted from the January 1996 to April 2010 records of the Department of Otolaryngology, Ninth People's Hospital and its Fengcheng Division, Shanghai Jiao Tong University School of Medicine. During this period, 211 hospitalized patients were diagnosed with laryngeal carcinoma. Of these, 136 patients were 60 years or older (64.5\%). In total, 122 patients were included in the study (117 men and 5 women). The median patient age was 68 years. Of the 122 patients (77.9\%), 95 had systemic comorbidities (Table 1): 52 patients had cardiovascular disease, 63 had pulmonary ventilation 
disorder and/or pulmonary insufficiency, 45 had diabetes mellitus, and 77 had hypertension. Other comorbidities such as prostatic hypertrophy had little effect on the patients' general condition and were therefore not considered in our analysis. All patients were pathologically diagnosed with squamous cell carcinoma of the larynx. Two patients underwent radiotherapy prior to surgical treatment.

Table 1. Systemic comorbidities in 122 patients with laryngeal carcinoma.
\begin{tabular}{lcccccc}
\hline Comorbidities & Any comorbidities & $\begin{array}{c}\text { Cardiovascular } \\
\text { disease }\end{array}$ & $\begin{array}{c}\text { Pulmonary } \\
\text { disease }\end{array}$ & Hypertension & Diabetes mellitus & $\begin{array}{c}\text { Two or more } \\
\text { comorbidities }\end{array}$ \\
\hline No. & 95 & 52 & 63 & 77 & 45 & 35 \\
Percentage (\%) & 77.9 & 42.6 & 51.6 & 63.1 & 36.9 & 28.7 \\
\hline
\end{tabular}

\section{Clinical classification of stage and $T$ grade}

Tumors were staged according to the TNM classification of the American Joint Committee on Cancer 2002 criteria. The distribution of cases by stage is presented in Table 2. No patients in the study cohort had subglottic cancers. Twenty-four patients had tumors that invaded 2-3 throat anatomy regions at the time of presentation. These cases were classified as cross-glottic because the primary tumor site could not be identified. In the entire cohort, stage III and IV lesions accounted for $67.2 \%(82 / 122)$ of cases and glottic cancers accounted for $59.8 \%(73 / 122)$ of cases, representing the most common clinical type.

\begin{tabular}{|c|c|c|c|c|c|c|c|c|c|}
\hline \multirow[t]{2}{*}{ Type } & \multirow[t]{2}{*}{ No. } & \multicolumn{4}{|c|}{$\mathrm{T}$ grade } & \multicolumn{4}{|c|}{ Stage } \\
\hline & & $\mathrm{T} 1$ & $\mathrm{~T} 2$ & T3 & $\overline{\mathrm{T} 4}$ & I & II & III & IV \\
\hline Supraglottic & 25 & 1 & 5 & 10 & 9 & 0 & 3 & 13 & 9 \\
\hline Glottic & 73 & 17 & 23 & 27 & 6 & 16 & 21 & 28 & 8 \\
\hline Cross-glottic & 24 & 0 & 0 & 14 & 10 & 0 & 0 & 11 & 13 \\
\hline Total & 122 & 18 & 28 & 51 & 25 & 16 & 24 & 52 & 30 \\
\hline
\end{tabular}

\section{Perioperative management}

The American Society of Anesthesiology (ASA) classification enables the preoperative estimation of the risk of anesthesia on the basis of the physical constitution of patients. Most patients were classified as having ASA stages 1 to 3,5 patients were classified as having ASA stage 4, and no patients were classified as having ASA stage 5. Two patients had degree III laryngeal obstruction at the time of presentation at the hospital. All patients with comorbidities completed relevant medical consultations, including at the departments of cardiology, respiratory care, and anesthesiology. These preoperative consultations were intended to assist in controlling the preexisting disease within the limit required for the performance of the operation. Patients were also monitored by heart physicians throughout the surgical procedure to reduce the incidence of stroke, which was an infrequent complication. After receiving systemic supportive treatment and conservative medical treatment, 5 patients with an ASA stage 4 physical condition transitioned to ASA stage 3 in approximately 1 week. Two patients who had 
degree III laryngeal obstruction underwent immediate tracheotomy combined with systemic conservative treatment to control the disease and stabilize their condition. Ten days later, their physical functioning assessment improved to ASA stage 3. After a comprehensive preoperative evaluation, it was decided that all 122 patients could tolerate operations performed under general anesthesia. The patients were continuously monitored for changes in comorbidities. Postoperatively, patients were managed with supportive therapy by parenteral nutrition.

\section{Appropriate surgical treatment}

The most suitable treatment strategy was determined on the basis of tumor stage, the patients' general condition, and the patients' preferences. Treatments included surgical operation alone, surgery combined with postoperative radiotherapy, surgery combined with chemotherapy, and surgery combined with both radiotherapy and chemotherapy. The basic principle of treatment selection was preservation of the normal tissue of the throat and larynx to ensure preservation of function, with complete tumor removal. A more detailed understanding of the tumor types, primary sites, morphologies, sizes, growth patterns, and differentiations that are found in elderly patients will help clinicians to select the most suitable therapeutic choices for this patient population, as will provide a better understanding of the general physical condition of elderly patients.

Tumor resection was performed according to the principle of "non-tumor" preservation, predominantly using a safety margin of $>5 \mathrm{~mm}$ and performing intraoperative frozen section pathological examination to verify the safety margin, except in a few cases of transoral semiconductor laser microsurgery. The patients were divided into 3 groups according to the surgical procedure performed (Table 3). Method 1 represented transoral semiconductor laser microsurgery for laryngeal resection. Method 2 represented partial laryngectomy, including cordectomy and three-quarters laryngeal resection with or without neck dissection. Method 3 represented total laryngectomy with or without neck dissection (single or bilateral). The excision of cervical lymph nodes was performed according to the criterium set at the Dalian meeting in 2004.

\begin{tabular}{|c|c|c|c|c|c|c|c|c|c|c|c|c|c|}
\hline \multirow[t]{2}{*}{ Surgical approach } & \multirow[t]{2}{*}{ No. } & \multicolumn{4}{|c|}{ Supraglottic } & \multicolumn{4}{|c|}{ Glottic } & \multicolumn{4}{|c|}{ Cross-glottic } \\
\hline & & $\mathrm{T} 1$ & $\mathrm{~T} 2$ & $\mathrm{~T} 3$ & $\mathrm{~T} 4$ & $\mathrm{~T} 1$ & $\mathrm{~T} 2$ & $\mathrm{~T} 3$ & $\mathrm{~T} 4$ & $\mathrm{~T} 1$ & $\mathrm{~T} 2$ & $\mathrm{~T} 3$ & $\mathrm{~T} 4$ \\
\hline Method 1 & 10 & - & - & - & - & 10 & - & - & - & - & - & - & - \\
\hline Method 2 & 25 & - & 1 & - & - & 5 & 17 & 2 & - & - & - & - & - \\
\hline Method 3 & 87 & 1 & 4 & 10 & 9 & 2 & 6 & 25 & 6 & - & - & 14 & 10 \\
\hline Total & 122 & 1 & 5 & 10 & 9 & 17 & 23 & 27 & 6 & - & - & 14 & 10 \\
\hline
\end{tabular}

\section{Repair of the laryngopharyngeal wound}

Patients who underwent transoral laser laryngeal resection did not require any repair, because the wound would heal spontaneously. For patients who underwent partial laryngectomy, the deficiency was initially repaired using an oral vestibular mucosa graft and fixed throat stent to expand the laryngeal cavity. However, because of poor tolerance postoperatively, the local larynx mucosa, pyriform sinus mucosa, outer membrane of the thyroid cartilage lamina, and the anterior strap muscles and their fascia were later used to perform repairs. Patients 
who underwent total laryngectomy and were more amenable to direct suture. For 2 of these patients, the pectoralis major myocutaneous flap was used to perform repairs because of tumors with wider invasion of the lateral pharyngeal wall and tongue root. For 3 of the patients who underwent total laryngectomy, additional esophageal resection was performed because of tumor invasion of the upper esophagus. In these cases, gastropharyngostomy involving gastric pull-up was performed.

\section{Statistical analysis}

Follow-up was ensured in a variety of forms, including clinical outpatient follow-up, telephone follow-up, and follow-up with family members. The last follow-up date in the present study was April 2010. Survival rates were calculated using the direct method, with persons lost to follow up classified as being dead.

\section{RESULTS}

In this study, restored pronunciation function was achieved for all patients who underwent Method 1 or 2 operations. Patients who underwent Method 3 operations practiced esophageal pronunciation, pronunciation assisted by handheld electronic aids, or body language to communicate with others. Level I postoperative wound healing was achieved in 114 cases (93.4\%). Level II and III healing accounted for 5 cases and 3 cases, respectively. Pharyngeal fistula in 2 cases ultimately healed, following 2 weeks of positive dressing changes and nutrition supportive therapy. No patients experienced postoperative difficulty in eating, and there were no cases of aggravation or deterioration of preexisting diseases during the perioperative period.

Over 5 years, 8 patients were lost to follow up. Survival rates for different categories of patients with laryngeal carcinoma are presented in Table 4. In the first year of follow-up, the follow-up rate was $100 \%$, the actuarial 1-year survival rate was $97.5 \%$ ( 3 patients died), and there were no statistically significant differences between survival rates for the various stages and types of carcinoma. At the end of the third year of follow up, the follow-up rate was $97.2 \%$ ( 3 cases were lost to follow up) and the actuarial 3 -year survival rate was $84.4 \%$ (14 patients had died). Again, there were no statistically significant differences between survival rates for the various stages and types of carcinoma. At the end of the fifth year of follow-up, the follow-up rate was $91.8 \%$ ( 8 cases were lost to follow up, including 5 since the third year of follow up), and the actuarial 5-year survival rate was $68.4 \%$ (23 patients had died). The 5-year actuarial survival rates differed significantly between patients with stage I and II tumors and those with stage III and IV tumors ( 80.6 and $61.3 \%$, respectively, $\chi^{2}=$ $3.91, \mathrm{P}<0.05)$. The 5-year survival rates did not differ significantly between patients with supraglottic and glottic tumors. However, the 5-year survival rates of patients with glottic and cross-glottic tumors were 75.8 and $47.4 \%$, respectively, constituting a statistically significant difference $\left(\chi^{2}=4.80, \mathrm{P}<0.05\right)$. The $1-, 3$-, and 5-year recurrence and metastasis rates were $4.1 \%(5 / 122), 17.4 \%(19 / 109)$, and $36.7 \%(36 / 98)$, respectively (these rates are calculated after excluding patients who were lost to follow up). Furthermore, of the 122 patients, 3 had second primary tumors: 1 had a gastric carcinoma, 1 had a renal carcinoma, and 1 had a tongue carcinoma. 
Table 4. Survival rate of 122 cases with laryngeal carcinoma (survival number/treatment number).

\begin{tabular}{|c|c|c|c|c|c|c|c|c|c|}
\hline \multirow[t]{2}{*}{ Clinical type } & \multicolumn{3}{|c|}{ 1-year survival rate } & \multicolumn{3}{|c|}{ 3-year survival rate } & \multicolumn{3}{|c|}{5 -year survival rate } \\
\hline & I+II stage & III+IV stage & Total & I+II stage & III+IV stage & Total & I+II stage & III+IV stage & Total \\
\hline Supraglottic & $3 / 3$ & $100(22 / 22)$ & $100(25 / 25)$ & $2 / 3$ & $89.5(17 / 19)$ & $86.4(19 / 22)$ & $2 / 3$ & $64.3(9 / 14)$ & $64.7(11 / 17)$ \\
\hline Glottic & $100(37 / 37)$ & $97.2(35 / 36)$ & $98.6(72 / 73)$ & $91.4(32 / 35)$ & $83.9(26 / 31)$ & $87.9(58 / 66)$ & $81.8(27 / 33)$ & $69.0(20 / 29)$ & $75.8(47 / 62)$ \\
\hline Cross-glottic & & $91.7(22 / 24)$ & $91.7(22 / 24)$ & & $71.4(15 / 21)$ & $71.4(15 / 21)$ & & $47.4(9 / 19)$ & $47.4(9 / 19)$ \\
\hline Total & $100(4 / 40)$ & $96.3(79 / 82)$ & $97.5(119 / 122)$ & $89.5(34 / 38)$ & $81.7(58 / 71)$ & $84.4(92 / 109)$ & $80.6(29 / 36)$ & $61.3(38 / 62)$ & $68.4(67 / 98)$ \\
\hline
\end{tabular}

\section{DISCUSSION}

The fastest growing element of the population comprises individuals older than 60 years. Furthermore, the proportion of deaths due to cancer has been increasing among elderly persons, especially in developing countries. Elderly patients with laryngeal carcinoma have their own particular characteristics. First, aging is a major risk factor for developing laryngeal tumors. However, symptoms of cancer are less evident in elderly patients, because of sensory hypofunction and frequent confusion of cancer symptoms with the symptoms of various preexisting diseases. These factors may result in misdiagnosis or delayed diagnosis. Indeed, in our study, stage III and IV tumors at the time of presentation accounted for $67.2 \%$ of cases. Second, the risk of surgery increases in elderly persons, in correspondence with age-related increases in multiple chronic systemic diseases (Peters et al., 2011). Of the patients in our cohort, $77.9 \%$ had 1 or more comorbidities. Therefore, thorough familiarity with the characteristics of elderly patients is essential, and efforts should be made to minimize the periods of disability and prolong the periods of health, if possible.

Because of age, systemic chronic diseases, and poor tolerance to surgery, elderly patients with laryngeal carcinoma often refuse surgical treatment. With the recent developments in geriatric medicine, anesthesiology, and throat surgery, elderly patients now require improvements in the quality of daily life, in addition to tumor removal and increased survival rates. Ageing itself is not a surgical contraindication, but general health status is (Molina et al., 2008). If physical functioning is relatively good and the lesions are amenable to operation in terms of extent, elderly patients can also undergo surgical therapy. Recent studies have found that aging is not a contraindication of laryngectomy with preservation of laryngeal function, but proper understanding of a variety of surgical indications and techniques is key to reducing postoperative complications in this patient population (Bridger et al., 1994; Beausang et al., 2003). Bumpous (1999) concluded that patients over the age of 80 years can also tolerate laryngectomy, if they are properly managed during the preoperative period. Sanders et al. (1990) suggested that supraglottic partial laryngectomy was well tolerated in elderly patients, provided that the individual was alert, was motivated to undergo swallowing rehabilitation, and had a speech therapist with whom to work. In this study, we identified 136 elderly patients with laryngeal carcinoma, of whom 122 received surgical treatment and 14 could not undergo surgery: 3 patients with a poor general condition who were not able to meet the requirements of ASA assessment despite appropriate care and were hence treated with other methods and 11 patients who with their families declined surgical treatment. Postoperative healing proceeded well for the 122 patients who underwent surgery, and there were no cases of aggravation or deterioration of comorbidities during the perioperative period. Careful evaluation of heart, lung, liver, and kidney function should be performed in elderly patients before laryngeal resection. 
Relevant medical departments should also be consulted in a timely fashion once problems have been detected. To particularly minimize perioperative and postoperative complications, close cooperation with the anesthesiologist is indispensable for the success of surgical interventions (Teymoortash et al., 2002). Anesthesiologists must determine the general condition of patients before surgery and decide the amount of anesthesia and anesthetic agents according to their physical status. Intraoperative adjustment of anesthetic drugs will prevent the occurrence of serious cardiovascular and cerebrovascular complications. Meanwhile, performance of the procedure by a skilled surgeon will minimize surgical trauma and shorten the operation time.

Furthermore, maintainance of adequate safety margins and performance of neck dissection are the key points for reducing recurrence and improving survival rates (Jose et al., 2003). As many studies have confirmed, the safety of cutting edge treatments is an important factor in patient prognoses (Spector et al., 2004; Vartanian and Kowalski, 2009). Lai et al. (2008) investigated 4 different therapies in patients with laryngeal carcinoma: surgery alone, surgery combined with postoperative radiotherapy, chemotherapy combined with radiotherapy, and surgery combined with chemotherapy. Their results showed that survival rates did not differ significantly between these various therapies. However, among patients undergoing postoperative radiotherapy, the survival rate with positive surgical margins was significantly lower than that with negative surgical margins (Lai et al., 2008). In a study of elderly patients with stage II or III laryngeal carcinoma, Fu et al. (2001) found that the 5-year survival rate among patients receiving postoperative radiotherapy was significantly higher than that among patients receiving radiotherapy alone. Fu et al. (2001) therefore suggested that failures in laryngeal carcinoma treatment mainly result from uncontrolled primary tumors and cervical lymph node metastases. In the present study, we ensured adequate safety margins $(\geq 5 \mathrm{~mm})$ during surgery. Further, we performed frozen section pathological examination to verify the safety margins during resection, which played an important role in improving the survival rates of patients.

In the present study, the 1-year actuarial survival rate was $97.5 \%$, which indicates that it is feasible to perform laryngectomy in elderly patients with laryngeal carcinoma. However, the 5 -year actuarial survival rate for patients with stage III and IV disease was significantly lower than that for patients with stage I and II disease. Accordingly, we believe that it is imperative to minimize morbidity and to render treatment with curative intent in the simplest manner possible for elderly patients with poorly controlled multiple medical problems. This conclusion is similar to the opinion of Bumpous (1999), who suggested that all treatment options that are available to younger patients should be available to healthy elderly patients but also that surgical treatment of the primary site and neck nodal disease with selective use of postoperative radiation therapy is preferred for elderly patients who are very ill.

Elderly patients with a compromised physical condition were treated with less aggressive surgical or radiation therapy, as compared with younger patients, in agreement with previous studies (Derks et al., 2005; Shiotani et al., 2010). Transoral $\mathrm{CO}_{2}$ laser microsurgery for cancer of the upper aerodigestive tract has played an increasingly important role in this aspect of oncology (Rudert and Werner, 1995; Werner et al., 2002). Its success is widely recognized and accepted. In recent years, semiconductor laser microsurgery using a self-retaining laryngoscope has been performed in patients with T1 laryngeal carcinomas and in some patients with T2 laryngeal carcinomas. We found that this method yielded good results and a significantly improved quality of life. Transoral semiconductor laser microsurgery is associated with less trauma, shorter treatment times, and relatively minor discomfort; it is a promising surgical 
approach for head and neck cancers, in general, and the majority of glottic and supraglottic laryngeal carcinomas, in particular.

This study provides further evidence that elderly laryngeal carcinoma patients should be treated according to their general condition and health, in conjunction with the specific subtype and stage of disease. Chronological age is itself no reason to treat elderly patients differently. However, we have only analyzed the effects of various laryngectomy surgeries on elderly patients; we have not considered other treatment options such as chemotherapy or cetuximab administration. According to a recent meta-analysis (Pignon et al., 2009), chemotherapy confers a significantly less benefit on elderly patients with head and neck cancers. Similarly, reductions in the benefits of cetuximab have also been observed in elderly patients (Bonner et al., 2010). Further studies should be conducted to investigate the outcomes of primary radiotherapy and chemotherapy or cetuximab treatment for laryngeal carcinoma in elderly patients. Such studies should attempt to identify optimal treatments for the each tumor stage, specifically focusing on elderly patients. In our research group, additional investigations will continue, including both a control study and a study that takes human papilloma virus infection into account.

In conclusion, the present study underscores the fact that elderly patients with laryngeal carcinomas should be treated with curative intent, and that age itself should never be the sole factor when selecting a therapeutic strategy for this disease. Presuming that adequate preoperative evaluations are performed and that operative indications are strictly controlled, surgery remains the primary means of treatment for elderly patients with laryngeal carcinoma.

\section{REFERENCES}

Barzan L, Veronesi A, Caruso G, Serraino D, et al. (1990). Head and neck cancer and ageing: a retrospective study in 438 patients. J. Laryngol. Otol. 104: 634-640.

Beausang ES, Ang EE, Lipa JE, Irish JC, et al. (2003). Microvascular free tissue transfer in elderly patients: the Toronto experience. Head Neck 25: 549-553.

Bhattacharyya N and Fried MP (2001). Benchmarks for mortality, morbidity, and length of stay for head and neck surgical procedures. Arch. Otolaryngol. Head Neck Surg. 127: 127-132.

Bonner JA, Harari PM, Giralt J, Cohen RB, et al. (2010). Radiotherapy plus cetuximab for locoregionally advanced head and neck cancer: 5 -year survival data from a phase 3 randomised trial, and relation between cetuximab-induced rash and survival. Lancet Oncol. 11:21-28.

Bridger AG, O'Brien CJ and Lee KK (1994). Advanced patient age should not preclude the use of free-flap reconstruction for head and neck cancer. Am. J. Surg. 168: 425-428.

Bumpous JM (1999). Treatment of stage III and IV supraglottic carcinoma: should elderly patients undergo standard treatment protocols? Arch. Otolaryngol. Head Neck Surg. 125: 1402-1404.

Clayman GL, Eicher SA, Sicard MW, Razmpa E, et al. (1998). Surgical outcomes in head and neck cancer patients 80 years of age and older. Head Neck 20: 216-223.

Derks W, De Leeuw JR, Hordijk GJ and Winnubst JA (2003). Elderly patients with head and neck cancer: short-term effects of surgical treatment on quality of life. Clin. Otolaryngol. Allied Sci. 28: 399-405.

Derks W, De Leeuw JR, Hordijk GJ and Winnubst JA (2005). Reasons for non-standard treatment in elderly patients with advanced head and neck cancer. Eur. Arch. Otorhinolaryngol. 262: 21-26.

Fu MN, Fand QL and Wang GM (2001). An Analysis of the treatment of 50 elderly patients with laryngeal carcinoma. $J$. Chin. Clin. Med. 2: 19.

Greenfield S, Blanco DM, Elashoff RM and Ganz PA (1987). Patterns of care related to age of breast cancer patients. JAMA 257: 2766-2770.

Jose J, Coatesworth AP, Johnston C and MacLennan K (2003). Cervical node metastases in squamous cell carcinoma of the upper aerodigestive tract: the significance of extracapsular spread and soft tissue deposits. Head Neck 25: 451-456.

Kowalski LP, Alcantara PS, Magrin J and Parise JO (1994). A case-control study on complications and survival in elderly patients undergoing major head and neck surgery. Am. J. Surg. 168: 485-490. 
Lai FY, Zhang Q, Guo ZM, Zeng ZY, et al. (2008). Treatment and prognosis of stage IV glottic laryngeal cancer. Ai Zheng. 27: 71-74.

Molina MA, Cheung MC, Perez EA, Byrne MM, et al. (2008). African American and poor patients have a dramatically worse prognosis for head and neck cancer: an examination of 20,915 patients. Cancer 113: 2797-2806.

Peters TT, van der Laan BF, Plaat BE, Wedman J, et al. (2011). The impact of comorbidity on treatment-related side effects in older patients with laryngeal cancer. Oral Oncol. 47: 56-61.

Pignon JP, le Maître A, Maillard E and Bourhis J (2009). Meta-analysis of chemotherapy in head and neck cancer (MACHNC): an update on 93 randomised trials and 17,346 patients. Radiother. Oncol. 92: 4-14.

Rudert HH and Werner JA (1995). Endoscopic resections of glottic and supraglottic carcinomas with the CO2 laser. Eur. Arch. Otorhinolaryngol. 252: 146-148.

Sanders AD, Blom ED, Singer MI and Hamaker RC (1990). Reconstructive and rehabilitative aspects of head and neck cancer in the elderly. Otolaryngol. Clin. North Am. 23: 1159-1168.

Shiotani A, Tomifuji M, Araki K, Yamashita T, et al. (2010). Videolaryngoscopic transoral en bloc resection of supraglottic and hypopharyngeal cancers using laparoscopic surgical instruments. Ann. Otol. Rhinol. Laryngol. 119: 225-232.

Spector GJ, Sessions DG, Lenox J, Newland D, et al. (2004). Management of stage IV glottic carcinoma: therapeutic outcomes. Laryngoscope 114: 1438-1446.

Teymoortash A, Wulf H and Werner JA (2002). Head and neck cancer surgery in the elderly. Laryngorhinootologie 81: 293-298.

Vartanian JG and Kowalski LP (2009). Acceptance of major surgical procedures and quality of life among long-term survivors of advanced head and neck cancer. Arch. Otolaryngol. Head Neck Surg. 135: 376-379.

Werner JA, Dunne AA, Folz BJ and Lippert BM (2002). Transoral laser microsurgery in carcinomas of the oral cavity, pharynx, and larynx. Cancer Control 9: 379-386.

Yang L, Parkin DM, Ferlay J, Li L, et al. (2005). Estimates of cancer incidence in China for 2000 and projections for 2005. Cancer Epidemiol. Biomarkers Prev. 14: 243-250. 